\title{
Real-Time Mamdani-type Fuzzy \\ Maneuvering Control of a Differential-Drive Mobile Robot
}

\author{
Thiago de A. Ushikoshi ${ }^{*}$ Rafael C. do Nascimento* \\ Pedro H. S. Coutinho ${ }^{* *}$ Thiago P. Chagas * \\ Leizer Schnitman ${ }^{* * *}$ \\ * Laboratório de Mecatrônica, Universidade Estadual de Santa Cruz, \\ BA, (e-mails: thiagoushikoshi@gmail.com, rafacardoso8@hotmail.com, \\ tpchagas@uesc.br). \\ ** Programa de Pós-Graduação em Enhenharia Elétrica, Universidade \\ Federal de Minas Gerais, MG, (e-mail: phcoutinho@ufmg.br). \\ *** Programa de Pós-Graduação em Mecatrônica, Universidade Federal \\ daBahia,BA, (e-mail: leizer@ufba.br).
}

\begin{abstract}
This paper concerns the real-time application of Mamdani-type Fuzzy Logic Controllers (FLCs) for maneuvering trajectory tracking of a differential-drive mobile robot, the Quanser QBot 2. Based on a recent fuzzy control strategy that allows the robot to move in both forward and backward directions (FB-Controller), a new FLC with sigmoidal and pi-shaped input membership functions is proposed, the FBm-Controller. The aim of this modification is providing smoother movements during the trajectory tracking, improving real-time performance. The proposal is compared with the FB-Controller and F-Controller, where the latter only allows forward movements. Simulations and real-time experiments performed on QBot 2 robot illustrate the advantages of FBm-Controller over the two other approaches.
\end{abstract}

Keywords: Fuzzy control, Maneuvering trajectory tracking, Differential-drive mobile robot, Dynamic modelling.

\section{INTRODUCTION}

Differently from binary logic systems, where the truthvalue of a proposition can only assume truth or false, fuzzy logic systems can admit intermediate truth-values. These are expressed linguistically, e.g., true, very true, false, very false, so that each linguistic term is related to a fuzzy set defined by a membership function (MF) which admits values within the real interval between 0 and 1 . Therefore, a progressively truth-value measure can be introduced for analysis (Zadeh, 1965).

Over the past decades, fuzzy logic has been applied in different areas: artificial intelligence, robotics, smart control systems and others. More specifically, fuzzy logic has also been successfully applied to control systems (Ross, 1995). The first fuzzy control approaches were based on Mamdani-type fuzzy logic controllers (FLC). Generally, FLCs have three stages: fuzzification, inference and defuzzification (Guerra et al., 2015). Based on that, a set of fuzzy relations maps the given inputs, for example the state variables, into a suitable control signal. Other approaches based on Takagi-Sugeno fuzzy systems were developed later, bringing advantages in the formal stability analysis based on the Lyapunov theory (Nguyen et al., 2019).

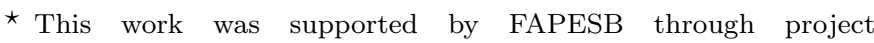
RED0004/2014.
}

The main advantage of Mamdani-type FLCs is their ability of incorporating human experience into the control scheme of complex processes with unknown or inaccurate model. The operator's knowledge is translated into a set of linguistic variables related by the fuzzy rules, allowing the incorporation of human experience, intuition or heuristics. With this tool, several processes can be appropriately stabilized without requiring precise or any mathematical model (Zimmermann, 2011). For example, in Wang et al. (2018), an adaptive FLC was proposed to perform output tracking of nonlinear systems with completely unknown dynamics.

Autonomous wheeled mobile robots have a wide range of applicability from manufacturing and security to aerospace and logistics (Peixoto et al., 2018). In particular, the trajectory tracking control is a basic task to be solved for this class of robots. Recently, different strategies have been proposed to deal with this problem, such as sliding mode control (Mija et al., 2016), fault-tolerant control (Yazdjerdi and Meskin, 2017) and fractional order control (RojasMoreno and Perez-Valenzuela, 2017). Within this context, FLCs have also been applied in the last years, such as fuzzy-PD control (Alouache and Wu, 2018) and optimized FLC with Particle Swarm Optimization (Nurmaini and Setianingsih, 2018).

In Ushikoshi et al. (2018), a new Mamdani-type fuzzy control strategy, called FB-controller, was proposed for 
maneuvering trajectory of a nonholonomic differentialdrive mobile robot (DDMR). It allows the robot moving in both forward and backward directions. It was compared with another control scheme that only allows forward movements, called F-controller. One of the contributions of this paper is applying the controllers developed in Ushikoshi et al. (2018) on real-time tests using the Quanser QBot 2 robot (Huq et al., 2009). In addition, as a second contribution, a new FLC with Sigmoidal and Pi MFs is proposed here, the FBm-Controller, which is also tested in the QBot 2. The aim of the proposed controller is providing smoother movements for the DDMR while reducing the trajectory tracking error and the control effort.

Although unnecessary for Mamdani-FLC adjustment, the DDMR model was used in Ushikoshi et al. (2018) to refine the controller tuning. Here, it is used for tuning, allowing it to be performed in a simulation environment and also allowing a numerical comparison between simulation's and real-time trajectories. A crossed-zigzag trajectory is considered to compare the performance among the three Mamdani-type FLCs. The comparison is made in terms of trajectory tracking error, control effort and also robot bouncing (linear velocity oscillation).

This paper is organized as follows. In Section 2, the DDMR modeling is presented, including kinematic and dynamic models, as well as the main specifications of Quanser QBot 2. In Section 3, the controllers studied in Ushikoshi et al. (2018) are revisited and the proposed FBm-Controller is presented. The methodology for simulation and real-time experiments are presented in Section 4. The performed simulation and real-time experiments results are presented in Section 5, where the advantages of FBm-Controller are highlighted. The conclusions and future directions are drawn in Section 6 .

\section{DIFFERENTIAL DRIVE MOBILE ROBOT}

Although Mamdani-type fuzzy systems may not require model, the mathematical model can be useful to test the fuzzy controller and eventually support modifications on parameters. In this section, kinematic and dynamic models of a DDMR are presented. For brevity, detailed mathematical development is omitted (see Ushikoshi et al. (2018) for details).

\subsection{Kinematic Model}

A general DDMR scheme with the robot and global frames is shown in Figure 1.

The system vector state is defined as $q=\left[\begin{array}{llll}x_{a} & y_{a} & \theta & \varphi_{r} \\ \varphi_{l}\end{array}\right]^{\top}$, where $x_{a}$ and $y_{a}$ are position coordinates of the centerpoint $A$ in the global frame, $\theta$ is the robot orientation and $\varphi_{r}$ and $\varphi_{l}$ are, respectively, the right and left wheel angular positions. These variables are also illustrated in Figure 1. Relating the DDMR linear and angular velocities to its wheels speeds in the global frame, the kinematic model is given as

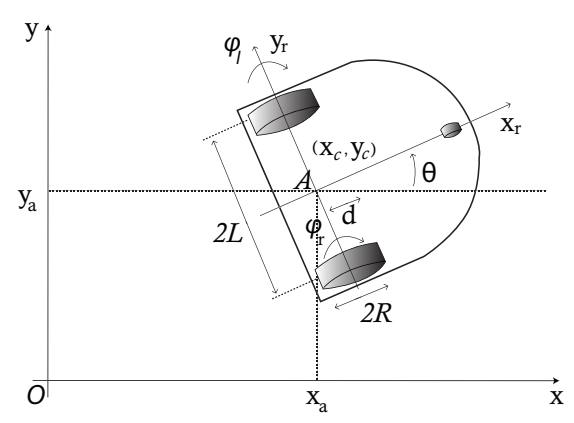

Figure 1. DDMR illustration. The subscripts $r$ and $l$ stands for right and left. Adapted from (Dhaouadi and Hatab, 2013).

$$
\dot{q}=\left[\begin{array}{cc}
\frac{R}{2} \cos (\theta) & \frac{R}{2} \cos (\theta) \\
\frac{R}{2} \sin (\theta) & \frac{R}{2} \sin (\theta) \\
\frac{R}{2 L} & -\frac{R}{2 L} \\
1 & 0 \\
0 & 1
\end{array}\right] \eta, \quad \eta=\left[\begin{array}{c}
\dot{\varphi}_{r} \\
\dot{\varphi}_{l}
\end{array}\right]
$$

where $\eta$ is the reduced state vector and $R$ and $L$ are robot dimensions according to Figure 1. More details can be found in Dhaouadi and Hatab (2013).

\subsection{Dynamic Model}

The DDMR dynamic model includes inertia, motor reduction and friction. Defining motor torque $\tau$ as input, the dynamics can be described using Lagrange method as follows (Dhaouadi and Hatab, 2013)

where

$$
\bar{H}(q) \dot{\eta}+\bar{V}(q, \dot{q}) \eta=\tau,
$$

$$
\begin{gathered}
\tau=\left[\begin{array}{l}
\frac{K_{t}}{R_{a}} v_{a r}-\left[\frac{K_{t}^{2}}{R_{a}}+b\right] \dot{\varphi}_{r} \\
\frac{K_{t}}{R_{a}} v_{a l}-\left[\frac{K_{t}^{2}}{R_{a}}+b\right] \dot{\varphi}_{l}
\end{array}\right] \\
\bar{H}(q)=\left[\begin{array}{cc}
I_{w}+\frac{R^{2}}{4 L^{2}}\left(m L^{2}+I\right) & \frac{R^{2}}{4 L^{2}}\left(m L^{2}-I\right) \\
\frac{R^{2}}{4 L^{2}}\left(m L^{2}-I\right) & I_{w}+\frac{R^{2}}{4 L^{2}}\left(m L^{2}+I\right)
\end{array}\right], \\
\bar{V}(q, \dot{q})=\left[\begin{array}{cc}
0 & \frac{R^{2}}{2 L} m_{c} d \dot{\theta} \\
-\frac{R^{2}}{2 L} m_{c} d \dot{\theta} & 0
\end{array}\right],
\end{gathered}
$$

$K_{t}$ is the torque constant, $R_{a}$ is the armature resistance, $v_{a}$ is the armature voltage, $b$ is the viscous friction constant, $m=m_{c}+2 m_{w}$ is the robot total mass, $m_{c}$ is the core's mass, $m_{w}$ is the wheel mass, $I_{w}$ is the wheels inertia, $I=I_{c}+m_{c} d^{2}+2 m_{w} L^{2}+2 I_{m}$ is the total equivalent inertia, $I_{c}$ is the core's inertia and $I_{m}$ is the motors inertia.

The following nonlinear friction equation is also introduced on the model (Makkar et al., 2005): 


$$
F=\left[\begin{array}{c}
F_{s, r} \tanh \left(\alpha_{s} \dot{\varphi}_{r}\right)-F_{k, r} \tanh \left(\alpha_{k} \dot{\varphi}_{r}\right) \\
F_{s, l} \tanh \left(\alpha_{s} \dot{\varphi}_{l}\right)-F_{k, l} \tanh \left(\alpha_{k} \dot{\varphi}_{l}\right)
\end{array}\right] m g,
$$

where $F_{s, r}$ and $F_{s, l}$ are static friction coefficients for right and left wheels respectively, $F_{k, r}$ and $F_{k, l}$ are the difference between static and kinetic friction coefficients for right and left wheels respectively, $\alpha_{s}$ and $\alpha_{k}$ are constants that determine how fast the tanh saturates and $g$ is the gravity acceleration. The friction produces a torque that opposes to $\tau$ in $(2)$.

\subsection{Experimental Platform: Quanser QBot 2}

The Quanser QBot 2, shown in Figure 2, is a nonholonomic ground robot equipped with embedded sensors and computer vision system. The communication between computer and robot is performed through a Data Acquisition Card by wireless connection. This communication is done by the Gumstix DuoVero, an embedded system responsible to process the software and command the actuators (Quanser, 2015). The code developed in Matlab/Simulink is sent to QBot 2. Then, the robot executes the software and return status and parameters to the central computer.

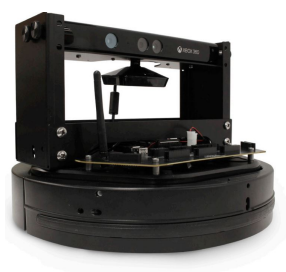

Figure 2. Quanser QBot 2. Extracted from Quanser (2015).

The system parameters are the following: The total mass is $m_{c}=3.79 \mathrm{~kg}$ (Quanser, 2015). Other parameters are estimated based on available commercial values: the wheel mass $m_{w}=1.72 \cdot 10^{-1} \mathrm{~kg}$; and wheel radius $R_{w}=$ $3.4 \cdot 10^{-2} \mathrm{~m}$. From Kobuki User Manual (Iclebo, 2017) we obtain: armature resistance $R_{a}=1.56 \Omega$; armature inductance $L_{a}=1.51 \mathrm{mH}$ and torque constant $K_{t}=$ 10.913 N.m/A.

\section{FUZZY CONTROLLERS STRUCTURE AND TUNING}

In this section, the FLCs proposed in Ushikoshi et al. (2018) are revisited. Some design details are omitted for brevity but can be found in Ushikoshi et al. (2018). Also, a new FLC is proposed by modifying the FB-Controller aiming to obtain better practical results.

\subsection{FB-Controller}

The main controller proposed in Ushikoshi et al. (2018), called FB-Controller, is proposed for bidirectional maneuvering. It receives distance $(l)$ and angle $(\Phi)$ to the target point as inputs and computes linear $\left(v_{F}\right)$ and angular $(\omega)$ velocities to follow the reference trajectory. The interval which contains all values that a variable can assume is called universe of discourse (Wang, 1997). The universes of discourse defined for each input are $0 \leq l \leq 5 \mathrm{~m}$ and $-3.141 \leq \Phi \leq 3.141 \mathrm{rad}$, respectively. For the outputs are $-0.1 \leq v_{F} \leq 0.1 \mathrm{~m} / \mathrm{s}$ and $-0.5 \leq \omega \leq 0.5 \mathrm{rad} / \mathrm{s}$, since the proposed maneuverings require low speed. The MFs and inference rules are shown in Figure 3 and Table 1, respectively. In addition, the Mean of Maximum method is used for defuzzification.

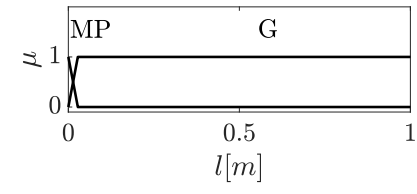

(a) Input $l$.

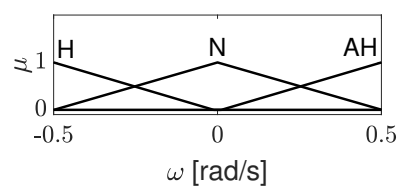

(c) Output $\omega$.

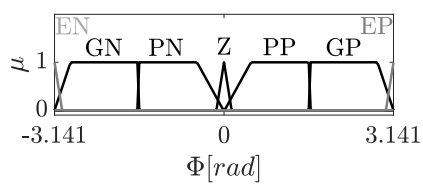

(b) Input $\Phi$.

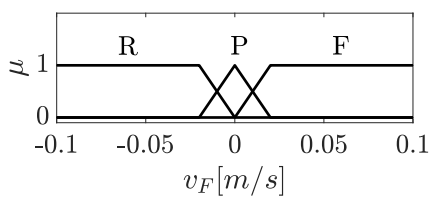

(d) Output $v_{F}$.
Figure 3. FB-Controller membership functions parameters.

Table 1. FB-Controller inference rules.

\begin{tabular}{|c|c|c|c|c|c|c|c|}
\hline \multirow{2}{*}{ Outputs } & \multicolumn{7}{|c|}{ Input } \\
\cline { 2 - 8 } & \multicolumn{7}{|c|}{$\Phi$} \\
\cline { 2 - 8 } & EN & GN & PN & Z & PP & GP & EP \\
\hline$v_{F}$ & $\mathrm{R}$ & $\mathrm{P}$ & $\mathrm{P}$ & $\mathrm{F}$ & $\mathrm{P}$ & $\mathrm{P}$ & $\mathrm{R}$ \\
\hline$\omega$ & $\mathrm{N}$ & $\mathrm{AH}$ & $\mathrm{H}$ & $\mathrm{N}$ & $\mathrm{AH}$ & $\mathrm{H}$ & $\mathrm{N}$ \\
\hline
\end{tabular}

\subsection{F-Controller}

For the sake of comparison, a controller which perform only forward movements, called F-Controller, was proposed in Ushikoshi et al.. Its inference rules are based on that shown in Table 1, excluding the possibility of backward movements. The MFs are the same as the FBController.

\subsection{FBm-Controller}

Here, a new controller called FBm-Controller is proposed. Aiming to obtain a smoother trajectory tracking, all MFs of $\Phi$ in Figure 3 are changed to Gaussian functions. The MFs of $v_{F}$ are changed to sigmoidal (sig) and pi-shaped. The MFs introduced in $\Phi$ are proposed as an alternative to create intermediate levels and, consequently, a smoother response. The universe of discourse of $v_{F}$ was changed to $-0.15 \leq v_{F} \leq 0.15 \mathrm{~m}$ and centroid method was used for defuzzification. The MFs of $\Phi$ and $v_{F}$ are illustrated in Figure 4 . The inference rules were created considering the same strategy as FB's and are shown in Table 2.
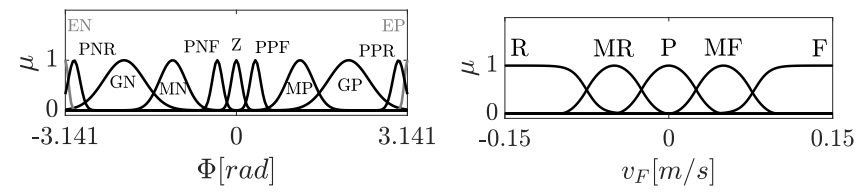

Figure 4. FBm-Controller membership functions parameters.

The following section presents the methodology used for application of the aforementioned controllers. 
Table 2. FBm-Controller inference rules.

\begin{tabular}{|c|c|c|c|c|c|c|c|c|c|c|c|}
\hline Outputs & \multicolumn{10}{|c|}{ Input } \\
\cline { 2 - 14 } & \multicolumn{10}{|c|}{$\mathbf{9}$} \\
\cline { 2 - 12 } & EN & PNR & GN & MN & PNF & Z & PPF & MP & GP & PPR & EP \\
\hline$v_{F}$ & R & MR & P & P & MF & F & MF & P & P & MR & R \\
\hline$\omega$ & N & AH & AH & H & H & N & AH & AH & H & H & N \\
\hline
\end{tabular}

\section{METHODOLOGY}

For the real-time application of the controllers, a computational model was developed in Simulink with QUARC on the host computer and downloaded to QBot 2 via wireless communication. A schematic block diagram of the model is shown in Figure 5. The main difference from Ushikoshi

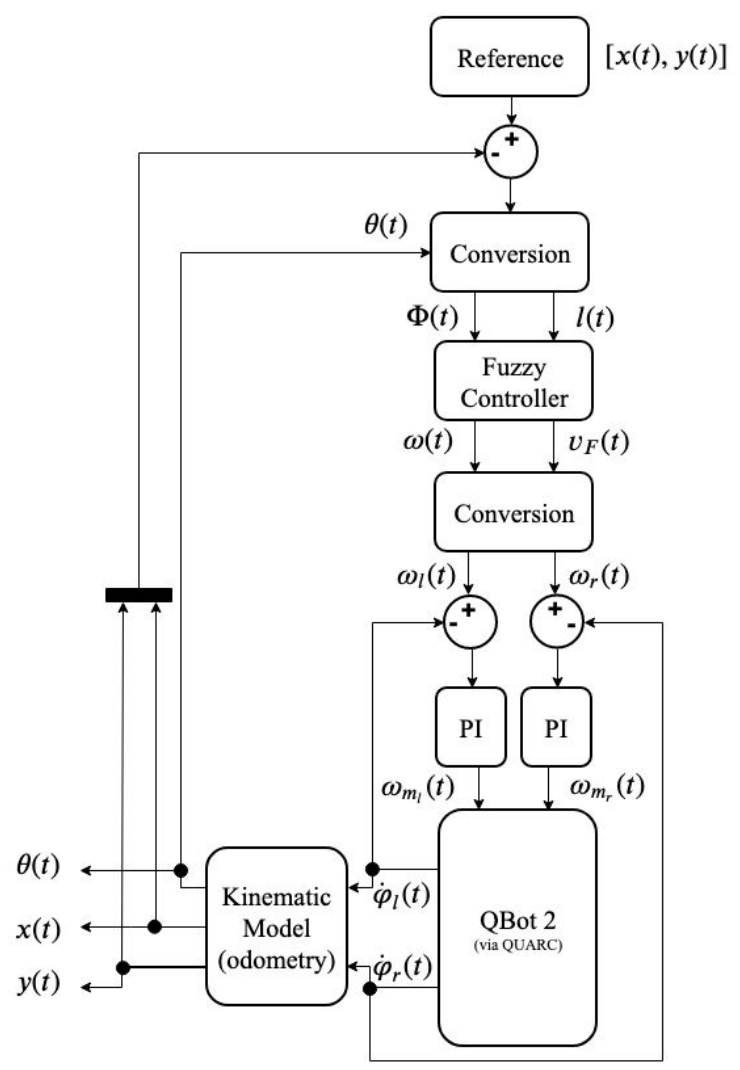

Figure 5. Schematic block diagram.

et al. (2018) is that the QBot 2 replaces the dynamic and actuators mathematical models presented in Section 2, but the kinematic model is used to estimate the robot localization through odometry. However, the gains of PI controllers used to control the motor speed of each wheel were modified to $K_{P}=2.5$ and $K_{I}=1$, avoiding robot bounces during motors acceleration. Besides, along with the universe of discourse boundaries, the computational model also includes armature voltage saturation of $12 \mathrm{~V}$. It is important to mention that although the PI controllers gains were modified, the fuzzy maneuvering controllers proposed by Ushikoshi et al. (2018) were applied without modification.

\section{SIMULATION AND REAL-TIME RESULTS}

In this section, the three controllers are evaluated by both numerical simulation and real-time application. Similar to Ushikoshi et al. (2018), a crossed zigzag (ZZ) trajectory is considered as case study. This trajectory is chosen as benchmark due to its sharp and smooth curves and straight lines. It is important to note that the trajectory setpoints include coordinates $x$ and $y$ are in meters.

The trajectory error $(\bar{e})$, computed using $\mathcal{L}_{2}$-norm, and control effort $\left(\bar{v}_{a}\right)$, computed using $\mathcal{L}_{1}$-norm, were chosen as performance indices ${ }^{1}$. They were calculated for both simulation and real-time tests and compared using absolute error. Also, as the linear velocity is monitored over time, other behavior features like quality and direction of motion can be compared as well.

The model developed in Matlab/Simulink powered by Quarc for application does not provide access to the voltage applied to the motors. Then, for a fair comparison, the control signal used to compute the control effort ${ }^{2}$ is defined as the linear velocities sent by the PI controllers to the robot.

\section{Case 1: FB-Controller}

The trajectories obtained by applying the FB-Controller to the mathematical model (simulation) and to the QBot 2 (real-time experiment) are shown in Figure 6(a). The arrow indicates the initial motion direction. Also, the time series for the robot center-point linear velocities are shown for both situations in Figure 6(b). The performance indices computed for this test are depicted in Table 2.

As shown in Figure 6(a), in both simulation and real-time applications, the robot tracked the trajectory accordingly. It is observed in Figure 6(a) and Table 2 that the FLC tuning is robust to real robot and model mismatch (notice that parametric errors are expected) and leads to equivalent results comparing simulation and real-time applications.

Some robot bouncing was observed during experiments applying FB-Controller and linear velocity oscillations noted in Figure 6(b) evidence this. Robot bouncing is undesirable and, although acceptable in small amplitude, one of the objectives of this paper is to propose a new controller to obtain smoother movements. In the sequel, FBm Controller results will be shown and a comparison to FB-Controller behavior shall be done.

Table 3. Performance indices for FBController.

\begin{tabular}{|c|c|c|}
\hline \multirow{2}{*}{ Test } & \multicolumn{2}{|c|}{ Performance indices } \\
\cline { 2 - 3 } & $\bar{e} \cdot 10^{-1}$ & $\bar{v}_{a} \cdot 10^{-1}$ \\
\hline Real-time & 0.641 & 1.374 \\
\hline Simulation & 1.168 & 0.860 \\
\hline Absolute error between tests & 0.527 & 0.514 \\
\hline
\end{tabular}

\section{Case 2: F-Controller}

The trajectories obtained using F-Controller are shown in Figure 7(a) and the robot linear velocities time series are

\footnotetext{
1 Unlike Ushikoshi et al. (2018), here the performance indices were divided by the number of samples so the comparison would be fair between trajectories with different number of setpoints.

2 In Ushikoshi et al. (2018) the control effort was computed based on the armature voltage applied to the motors.
} 


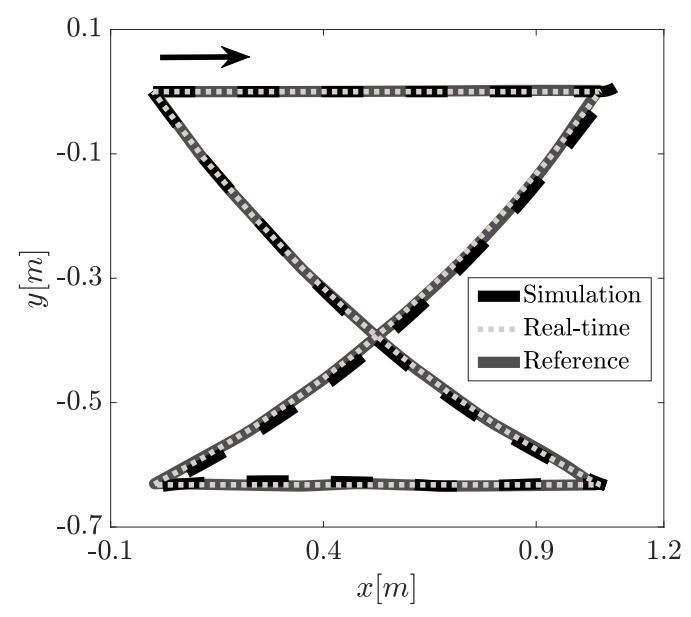

(a) Trajectories using FB-Controller.

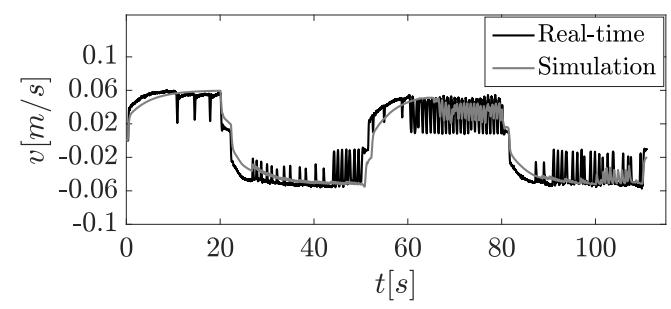

(b) Linear velocities using FB-Controller.

Figure 6. Trajectories and linear velocities performed in

FB-Controller real-time test and simulation.

shown in Figure 7(b). The performance indices computed for this case are depicted in Table 4.

Table 4. Performance indices for F-Controller case.

\begin{tabular}{|c|c|c|}
\hline \multirow{2}{*}{ Test } & \multicolumn{2}{|c|}{ Performance indices } \\
\cline { 2 - 3 } & $\bar{e} \cdot 10^{-1}$ & $\bar{v}_{a} \cdot 10^{-1}$ \\
\hline Real-time & 2.131 & 1.395 \\
\hline Simulation & 2.952 & 0.903 \\
\hline Absolute error between tests & 0.821 & 0.492 \\
\hline
\end{tabular}

The F-Controller was not capable to complete the given path in the real-time test nor in the simulation. This is in line with the FB-Controller advantages over the FController presented in Ushikoshi et al. (2018), where the FB-Controller could reduce the amplitude of the rotations once it uses bidirectional driving - reducing the time needed to complete the trajectory. Furthermore, the results in Table 4 shows that the absolute error between tests for the trajectory error was bigger than the previous case (Table 2). This could indicate increase of sensitivity of the controller to parametric uncertainty.

\section{Case 3: FBm-Controller}

The simulation and real-time trajectories obtained using the FBm-Controller are shown in Figure 8(a) and the robot linear velocities are shown in Figure 8(b). Table 5 shows the computed results for the performance indices and the absolute errors.

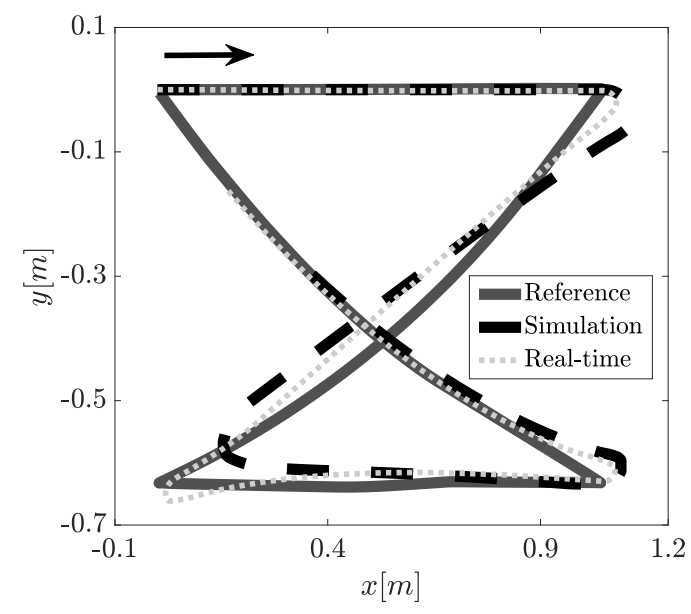

(a) Trajectories using F-Controller.

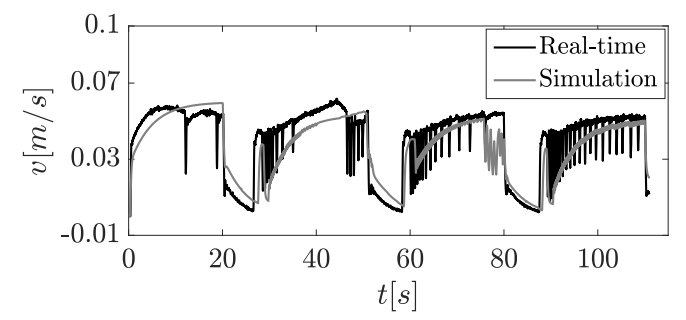

(b) Linear velocities using F-Controller.

Figure 7. Trajectories and linear velocities performed in F-Controller real-time test and simulation.

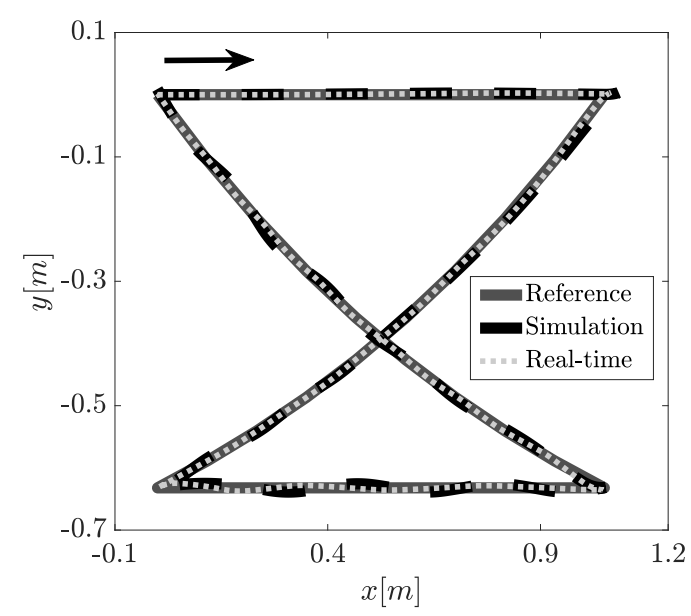

(a) Trajectories using FBm-Controller.

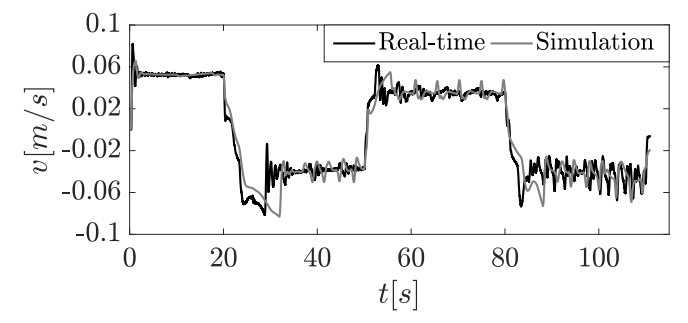

(b) Linear velocities using FBm-Controller.

Figure 8. Trajectories and linear velocities performed in FBm-Controller real-time test and simulation. 
Table 5. Performance indices for FBmController case.

\begin{tabular}{|c|c|c|}
\hline \multirow{2}{*}{ Test } & \multicolumn{2}{|c|}{ Performance indices } \\
\cline { 2 - 3 } & $\bar{e} \cdot 10^{-1}$ & $\bar{v}_{a} \cdot 10^{-1}$ \\
\hline Real-time & 0.316 & 1.029 \\
\hline Simulation & 0.458 & 0.903 \\
\hline Absolute error between tests & 0.142 & 0.126 \\
\hline
\end{tabular}

The FBm-Controller led the robot to track the given trajectory accordingly, as presented in Figure 8(a). Comparing to the FB-Controller, it provided a smoother control, as can be seen in Figure 8(b). The linear velocity oscillations and visual robot bouncing in this case are significantly smaller than those presented by the other controllers. One of the causes is the controller's proposition, where the MFs were modified to provide a smoother fuzzy surface - leading to smaller tracking error. As presented in Table 5, the absolute errors between simulation and real tests are the smallest among the analysed controllers. Also, the control efforts were very close between real-time and simulation tests. Comparing with previous cases, the FBm-Controller showed to be more robust to model mismatch.

\section{CONCLUSION}

This paper applied the $\mathrm{F}$ and $\mathrm{FB}$ controllers proposed by Ushikoshi et al. (2018) on real-time tests using the QBot 2 robot. Furthermore, the present paper proposed and also tested a new controller, called FBm-controller, aiming to obtain smoother robot motion. Results highlight the practical verification of the FB-Controller advantages over the F-Controller, as discussed in Ushikoshi et al. (2018). Besides, model mismatch has taken different proportions according to the controller used and robustness was discussed. The FBm-Controller led to smaller trajectory error and control effort, showing its effectiveness. In addition, the absolute errors between simulation and real-time application presented in this case were the smallest, showing better robustness to parameter uncertainties. Also, it led the robot to perform a smoother motion avoiding the robot bouncing, which is undesirable for several applications.

So it is reasonable to associate the smoother shape and composition of the MFs to its smoother decision criteria. Moreover, the model based tuning has proven it effectiveness, even more with the robustness inherent to FLCs. For future works, we suggest a model parameter identification applied to the nonlinear model described, for example using optimization. Also, is desirable performing stability and robustness analysis of Mamdani-type FLCs applied to DDMRs and also including direction $(\theta)$ control.

\section{REFERENCES}

Alouache, A. and Wu, Q. (2018). Fuzzy logic pd controller for trajectory tracking of an autonomous differential drive mobile robot (ie quanser qbot). Industrial Robot: An International Journal, 45(1), 23-33.

Dhaouadi, R. and Hatab, A.A. (2013). Dynamic modelling of differential-drive mobile robots using lagrange and newton-euler methodologies: A unified framework. Advances in Robotics \& Automation, 2(2), 1-7.
Guerra, T.M., Sala, A., and Tanaka, K. (2015). Fuzzy control turns 50: 10 years later. Fuzzy Sets and Systems, $281,168-182$.

Huq, R., Lacheray, H., Fulford, C., Wight, D., and Apkarian, J. (2009). Qbot: an educational mobile robot controlled in matlab simulink environment. In Electrical and Computer Engineering, 2009. CCECE'09. Canadian Conference on, 350-353. IEEE.

Iclebo (2017). Kobuki User Guide 1.1.0.

Makkar, C., Dixon, W., Sawyer, W., and Hu, G. (2005). A new continuously differentiable friction model for control systems design. In Advanced Intelligent Mechatronics. Proceedings, 2005 IEEE/ASME International Conference on, 600-605. IEEE.

Mija, S. et al. (2016). Robust controller for trajectory tracking of a mobile robot. In Power Electronics, Intelligent Control and Energy Systems (ICPEICES), IEEE International Conference on, 1-6. IEEE.

Nguyen, A.T., Taniguchi, T., Eciolaza, L., Campos, V., Palhares, R., and Sugeno, M. (2019). Fuzzy control systems: Past, present and future. IEEE Computational Intelligence Magazine, 14(1), 56-68.

Nurmaini, S. and Setianingsih, F. (2018). Enhancement of the fuzzy control response with particle swarm optimization in mobile robot system. In 2018 International Conference on Electrical Engineering and Computer Science (ICECOS). IEEE.

Peixoto, K.P., Coutinho, P.H.S., Araújo, A.C., Almeida, B.P.S., Souto, F.H.S., das Chagas, T.P., and Schnitman, L. (2018). Application of path planning based on artificial potential fields to mobile robots with distributed processing. In Anais do X Congresso Nacional de Engenharia Mecânica. ABCM.

Quanser (2015). QBOT 2 - User Manual. 2015.

Rojas-Moreno, A. and Perez-Valenzuela, G. (2017). Fractional order tracking control of a wheeled mobile robot. In Electronics, Electrical Engineering and Computing (INTERCON), 2017 IEEE XXIV International Conference on, 1-4. IEEE.

Ross, T. (1995). Fuzzy logic with engineering applications. McGraw-Hill.

Ushikoshi, T.A., Peixoto, K.P., Souto, F.H.S., das Chagas, T.P., and Schnitman, L. (2018). Fuzzy maneuvering controller applied to a dynamic model of a differential drive mobile robot. In 2018 IEEE International Conference on Fuzzy Systems (FUZZ-IEEE).

Wang, L. (1997). A Course in Fuzzy Systems and Control. Prentice Hall PTR.

Wang, N., Sun, J.C., and Er, M.J. (2018). Trackingerror-based universal adaptive fuzzy control for output tracking of nonlinear systems with completely unknown dynamics. IEEE Transactions on Fuzzy Systems, 26(2), 869-883.

Yazdjerdi, P. and Meskin, N. (2017). Fault tolerant control of differential drive mobile robots using sliding mode controller. In Control, Instrumentation, and Automation (ICCIA), 2017 5th International Conference on, 270-274. IEEE.

Zadeh, L.A. (1965). Fuzzy sets. Information and Control, $8(3), 338-353$.

Zimmermann, H. (2011). Fuzzy Set Theory and Its Applications. SpringerLink : Bücher. Springer Netherlands. 